\title{
Leader
}

\section{A practical approach to suspicious death in infancy-a personal view}

\author{
Michael A Green \\ "And it died, because she overlaid it" (I Kings 4, \\ 16-19)
}

This quotation, taken from the account of King Solomon's judgement in a case of disputed motherhood, is often cited as the first example of sudden infant death syndrome (SIDS). I view this claim with increasing scepticism. The child was only a few days old. Both the women involved were of a profession associated with "at risk" children. The child was left in the care of an unqualified minder, and one suspects that there were other visitors to the disorderly house as well. It is minor details such as these that should raise awareness in the mind of any pathologist called upon to investigate the death of an infant under 12 months old.

In an ideal world, all sudden and unexpected deaths in childhood would be investigated by paediatric pathologists, forensic pathologists with some paediatric experience, or ideally the two working as a team. I am mindful of Clothier's recommendations following the case of Nurse Beverley Allitt, ${ }^{1}$ but unconvinced by his insistence that a paediatric pathologist should investigate all such cases. In the foreseeable future it is unlikely that there will be sufficient paediatric or forensic pathologists to implement Clothier. The majority of these deaths will be referred to the coroner, procurator fiscal, or other appropriate law officer (or should be), but the necropsies will be performed by general hospital pathologists, many of whom - but by no means all - will have a declared interest in paediatric pathology.

Over the last few years there has been a dramatic fall in the incidence of SIDS. This improvement is owing to several factorsbetter antenatal care, better management of infants at risk by reason of prematurity or birth trauma, and better postnatal care, advice, and supervision. The "Back to Sleep" campaign which followed the death of the television presenter Anne Diamond's child, Sebastian, in

Forensic Pathology, University of Sheffield, The Medico-Legal Centre, Watery Street, Sheffield S3 7ES, UK M A Green

Correspondence to: Professor M A Green.

Accepted for publication 13 May 1998
1991 has produced the most dramatic fall. Similar campaigns in New Zealand, Australia, and the United States have also met with gratifying success. ${ }^{2}$

It is difficult to ascertain the exact number of SIDS-type deaths; in 1994 the figures from the Central Statistical Office for England and Wales were 331 , but rose by $6 \%$ in $1996 .^{3}$ There is currently a tendency among some pathologists, certainly in my own department, to classify an increasing number of these deaths as "not ascertained." It has always been suspected, although those suspicions have only been uttered in muted tones, that unlawful acts by parents or carers account for a substantial minority of sudden infant deaths. Wilczynski first brought the issue to prominence in $1994^{4}$ and expanded further upon the work in $1997 .{ }^{5}$ She reviewed the necropsy reports and surrounding circumstances of a large number of infant deaths, and estimated that the true figure for homicide in England and Wales under the age of one year could be of the order of 150 deaths a year. I take the view that she was overpessimistic, but I am certain that the true annual figures are considerably higher than the 50 to 80 claimed at different times by the Home Office, the NSPCC, and other concerned agencies over the last decade. If we also accept that the number of cases of proven deliberate harm to infants remains fairly constant from year to year, this means that, as the overall numbers of sudden infant deaths fall, a greater proportion of their total will be "suspicious," and will require very detailed investigation. A recent infant death necropsy protocol from Ontario, Canada, ${ }^{6}$ carried on its first page the message, printed in bold, "THINK DIRTY!," and advised all concerned to treat every so called SIDS death as a possible homicide until proved otherwise-a view which I strongly support. I accept that this is a minority opinion, strongly opposed by many coroners, pathologists, and even some police officers, but its implementation has brought unexpected cases to light.

There are now readily available detailed protocols for the necropsy examination of cases of sudden death in infancy (as provided, for example, by the Confidential Enquiry into Stillbirth and Deaths in Infancy (CESDI)).${ }^{78} \mathrm{I}$ do not propose to rehearse them here. I would merely emphasise and support the need for full histological examination (despite the coroners' inability, or in some cases reluctance, to pay), appropriate toxicology, microbiology, and biochemical investigations. However, I must stress that if there is any suspicion whatsoever that the child might have been harmed, vitreous humour should not be taken "blind" at the commencement of the examination. It may become far more important that the eyes be preserved for fixation and histological examin- 
ation. A reasonable compromise is only to take vitreous after the skull has been opened, the brain inspected, and the absence of any intracranial bleeding (or old staining of the dura) confirmed. Likewise, no attempts at transcutaneous extraction of heart blood, or cerebrospinal fluid by whatever route, should be made in such cases.

The problem which confronts the investigating pathologist is that child homicide is often subtle. A review of cases in the Sheffield Department's archives covering the last 30 years has shown that the classical "battered child" with multiple external injuries is now the exception rather than the rule. The only way to diagnose poisoning is to think of it. Deliberate upper respiratory obstruction produces very few signs, and the distinction between it and true SIDS is almost impossible.

The typical SIDS infant shows no external signs whatsoever. Some paediatric pathologists are prepared to accept the presence of facial petechiae in $2-3 \%$ of cases. ${ }^{9}$ My own experience suggests that this estimate may be overcharitable. Internal examination may reveal thymic petechiae, scattered coarse petechiae on the surfaces of the lungs, and occasional petechiae on the epicardial surface of the heart, particularly along the coronary sulci. The lungs are rather heavier than normal, and a small amount of parenchymal haemorrhage might be present to the naked eye and on microscopical examination of their cut surfaces.

In deliberate upper respiratory obstruction, external petechiae-whether confined to the face or more generalised-are seldom seen. Their presence, even in very small numbers, should immediately cause warning bells to ring. Upon internal examination there may be numerous petechiae in the thymus, the lungs show coarse subpleural petechiae in profusion, and their cut surfaces may be frankly bloody. ${ }^{10}$ Work currently in progress in Sheffield has led us to adopt the practice that in any case where intra-alveolar haemorrhage occupies more than $5 \%$ of the surface area of three microscopical lung sections randomly selected from those taken at necropsy, SIDS should not be given as the cause of death. The cause of death should be reported as "not ascertained" and appropriate discussions with the coroner and the police should be held. The commentary at the end of the necropsy report should set out in detail the explanation for this caution; the strength of the pathologist's suspicions, if suspicion there be, should be clearly indicated.

Of even more concern is the finding of haemosiderin laden macrophages when lung sections are stained with Perls' Prussian blue reagent. The presence of these suggests that previous episodes of deliberate upper respiratory obstruction might have occurred. Reports from New Zealand ${ }^{11}$ and studies in progress in Nottingham, Bristol, and Sheffield are attempting to shed further light on this problem. Such macrophages are sometimes present in the lungs of premature infants who die in the first few weeks of life, or in those who have undergone prolonged assisted respiration. I believe it unlikely that they persist at six months and beyond, but further studies are needed.

What therefore should pathologists do when confronted with cases of sudden death in infants? A detailed history is essential. The two line, hand written "sudden death report" or telephone message from the coroner's office is entirely inadequate. SIDS, by internationally accepted definition, can occur up to the age of 12 months. In my view, any death outside the period eight to 14 weeks should be regarded as unusual, as should a previous history of near fatal collapses or "blue attacks" during the daytime, especially if associated with feeding difficulties, and witnessed only by the mother. I view any daytime death with some scepticism, particularly if the child was allegedly well only an hour or so before.

The child's previous medical history is important. ${ }^{12}$ Prematurity and the resulting separation from the mother if the baby was admitted to a neonatal unit may have led to a lack of bonding. Histories of "failure to thrive," diarrhoea, or dubious food allergies are of serious prognostic significance. If the child has had previous hospital admissions for such conditions, for "asthma," or unusual skin rashes, the hospital case notes should be obtained and particular attention should be paid to the development charts, particularly if weight gain lags behind growth. Miraculous weight gains upon admission to hospital indicate that there is something very wrong at home.

Details of the parents and carers should also be taken into consideration. Meadow has published widely on "Munchausen syndrome by proxy" and similar conditions. ${ }^{13}$ The mothers liable to suffer from this distressing disorder, who inflict harm upon their children to gain attention for themselves but do not intend to cause death, often show distinctive factors in their own history. They may have been abused themselves as children, often have records of teenage violence or eating disorders, have had multiple partners, and may indeed have been mother or carer to a previous so called "cot death." A history of self harm, drug abuse, or petty crime is also significant. Members of the caring professions who have access to drugs and have some medical and pharmaceutical knowledge should also be subjected to close scrutiny. Deliberate upper respiratory obstruction was underappreciated and underdiagnosed until the pioneering surveillance work of Southall ${ }^{12}$ in Staffordshire and Byard ${ }^{2}$ in South Australia. Retrospective reviews of family histories by Meadow and others have also heightened our awareness of this condition.

After the necropsy, including ancillary investigations, has been completed, the results (or lack of them) usually raise more questions than answers. All too commonly, several of the risk factors outlined above are present. The degree of pulmonary parenchymal haemorrhage is such that SIDS should not be given as the cause of death. Under no circumstances should a pathologist ever go into the witness box and say dogmatically "this is smothering." The best outcome that one can hope for after giving the 
cause of death as "not ascertained" is that, through the coroner, social services will be made aware of the circumstances. Any future child borne into the family will be closely supervised. My own practice in preparing the report to the coroner is to write a clear explanation of my reasons for giving a nonspecific cause of death. If I have concerns or suspicions, I record those in my conclusions, albeit in guarded tones. I also may write directly to the family doctor and the Director of Social Services-with the prior agreement of the coroner of course!

I see no prospect of hard and fast solutions to these difficult cases. Bacon ${ }^{14}$ has recently proposed alternative mechanisms for the investigation of sudden infant death, but we are unlikely to see any changes in the legal framework which constrains the present system within the foreseeable future. The results of the current studies described above are awaited with great interest. In the meantime, I urge the more frequent use of "Not ascertained" as a cause of death. I accept unreservedly that this practice can cause distress and offence to blameless recently bereaved parents. However, I hold a strong personal belief that there is a duty upon the pathologist to draw that attention of the authorities to any unusual findings rather than to take the easy way out. The performance of a necropsy on a subse- quent child, in whom the evidence of homicide is clear, is an unforgettable and salutary experience.

1 Clothier C. The independent enquiry relating to death and injuries on the children's ward at Grantham and Kesteven General Hospital during the period February to April 1991. London: HMSO, 1994.

2 Byard RW. Sudden infant death syndrome-a diagnosis in search of a disease. 7 Clin Forensic Med 1995;2:121-8.

3 Safety plea to parents as cot deaths rise. The Independent 1997 Aug 28.

4 Wilczynski A. The incidence of child homicide: how accurate are the official statistics? F Clin Forensic Med 1994; 1:61-6.

5 Wilczynski A. Child homicide. London: Greenwich Medical Media, 1997:35-6.

6 Protocol for the investigation of sudden and unexpected Protocol for the investigation of sudden and unexpected
deaths in children under 2 years of age [Memorandum No 631]. Ontario: Ministry of the Solicitor General and 631]. Ontario: Ministry of
Correctional Services, 1995.

7 National Advisory Body for Confidential Enquiry into Stillbirth and Deaths in Infancy. Third annual report. London: Department of Health, 1996.

8 Royal College of Pathologists. Minimum guidelines for post-mortem investigation of post neonatal infant deaths and sudden deaths in infancy. In: Guidelines for post mortem reports. London: Royal College of Pathologists, 1993: appendix 1.7.10.

9 Berry PJ. Pathological findings in SIDS. F Clin Pathol 1992; 45(suppl):11-16.

10 Kleeman WJ, Wiechern V, Schuck M. Intrathoracic and subconjunctival petechiae in sudden infant death syndrome. Forensic Sci Int 1995;72:49-54.

11 Becroft DMO, Lockett BK. Intra-alveolar pulmonary siderophages in sudden infant death: a marker for siderophages in sudden infant death: a marker
previously imposed suffocation. Pathology 1997;29:60-3.

12 Poets CF, Southall DP. Recent developments in research into sudden infant death. Thorax 1994;49:196-7.

13 Meadow SR. Munchausen syndrome by proxy. Med Leg 7 1995;63:89-104.

14 Bacon CJ. Cot death after CESDI. Arch Dis Child 1997;76: 1271-3. 